\title{
Bewegte Zeiten - Zwischen autonomer Zeitplanung, Planungssicherheit und Flexibilität
}

\author{
Anna Arlinghaus ${ }^{1}$ Johannes Gärtner ${ }^{1}$ Sylvia Rabstein ${ }^{1} \cdot$ Sebastian Schief ${ }^{1}$ Anne Marit Wöhrmann'
}

๑ Springer-Verlag GmbH Deutschland, ein Teil von Springer Nature 2019

Liebe Leserinnen, liebe Leser,

der Europäische Gerichtshof in Luxemburg hat entschieden, dass die Mitgliedstaaten der EU zukünftig Arbeitgeberinnen und Arbeitgeber zur Arbeitszeiterfassung ihrer Beschäftigten verpflichten müssen. Gleichzeitig fordern sowohl Unternehmen wie Beschäftigte Flexibilisierung und Anpassung an die Möglichkeiten des modernen Arbeitens und der Digitalisierung. Nationale gesetzliche Arbeitszeitenregelungen werden - wie z.B. in Österreich - gelockert und zur Diskussion gestellt. Bewegte Zeiten also für Arbeitszeitforscherinnen und -forscher und alle, die sich mit der Gestaltung von Arbeitszeit auseinandersetzen.

Den unterschiedlichen Aspekten der Flexibilisierung widmeten sich die Beiträge des 6. Symposiums der Arbeitszeitgesellschaft in den Räumen der Bundesanstalt für Arbeitsschutz und Arbeitsmedizin (BAuA) am 5. Oktober 2018 in Dortmund. Unter dem Titel „Individuell, flexibel und digital - Arbeitszeit 4.0 in Wissenschaft und Praxis" wurde dabei ebenso auf Digitalisierung wie auch auf Aspekte wie Entgrenzung, Pendeln, Vereinbarkeit von Familie und Beruf, Selbstbestimmtheit, Big Data oder Aspekte der Arbeitsgestaltung bei Schichtarbeitenden eingegangen. Das vorliegende Themenheft präsentiert eine Auswahl der auf dem Symposium diskutierten Beiträge.

Die Rolle von Zeiterfassung und variablen Arbeitszeiten wird in zwei Beiträgen thematisiert. So untersuchten Astleithner und Stadler die Auswirkungen selbstgesteuerten Arbeitens ohne Zeiterfassung auf die Länge der Arbeitszeiten. Für die Beantwortung der Frage eines möglichen Zusammenhangs zwischen Zeiterfassung und Arbeitslänge verwenden die AutorInnen umfangreiche Daten der Amtlichen Statistik in Österreich. Ihre Ergebnisse zeigen, dass Arbeitszeiten vor allem bei hochgradig selbstgesteuerter Arbeit länger sind. Nachreiner, Arlinghaus und Greubel

\footnotetext{
Dr. Phil. Anna Arlinghaus

arlinghaus@ximes.com

1 XIMES GmbH, Hollandstraße 12/12, 1020 Wien, Österreich
}

analysierten Daten des European Working Conditions Survey 2010 und beobachteten erhöhte Risikoschätzungen für Arbeitsunfälle in Verbindung mit sehr variablen Arbeitszeiten.

Im Beitrag von Seitz werden Analysen zu Arbeitszeitautonomie und sogenanntem Leader-Member-Exchange im Hinblick auf Stress vorgestellt. Anhand einer Befragung von in Vertrauensarbeitszeit Beschäftigten wird hier deutlich, dass eine vertrauensvolle Beziehung zur Führungskraft sowie Arbeitszeitautonomie helfen können, die spezifischen Arbeitsanforderungen innerhalb der Vertrauensarbeitszeit zu bewältigen.

Ausreichende Pausen- und Ruhezeiten können eine Ressource zur Verringerung belastender Arbeitsbedingungen darstellen. Fallen diese jedoch aus oder sind unzureichend lang, können Risiken entstehen, die in zwei Beiträgen betrachtet werden: Backhaus, Brauner und Tisch behandeln in ihrem Beitrag die Auswirkungen verkürzter Ruhezeiten von Vollzeitbeschäftigten in der BAuA-Arbeitszeitbefragung 2017. Sie zeigen darin, dass die Verkürzung von Ruhezeiten mit vermehrten psychosomatischen Beschwerden und schlechterer Work-Life-Balance assoziiert ist. Lohmann-Haislah et al. betrachten den Ausfall gesetzlicher Ruhepausen in der BIBB/BAuA-Erwerbstätigenbefragung 2012. Sie berichten, dass Pausen häufiger im Pflegebereich als in anderen Bereichen ausfallen und auch das Beschäftigungsverhältnis eine Rolle spielt. Beschäftigte mit einem unbefristeten Vertrag ließen in dieser Befragung häufiger die Pause ausfallen.

Ein Praxisbeispiel zum Thema der Umsetzung von Empfehlungen und Regelungen zur Verbesserung der Gesundheit von Beschäftigten durch eine arbeitswissenschaftlich empfohlene Schichtplangestaltung wird von Watrinet et al. vorgestellt. Der Beitrag beschreibt das Vorgehen sowie zentrale Ergebnisse der Arbeitsgruppe „Gesünderes Arbeiten in der Polizei“, die in Rheinland Pfalz im Jahr 2015 gebildet wurde.

Soziale Auswirkungen von Schichtarbeit werden in dem Beitrag von Müller und Lück im Hinblick auf die Vereinbarkeit von Beruf und Privatleben behandelt. Ausgehend von 
Daten der BIBB/BAuA-Erwerbstätigenbefragung aus dem Jahr 2018 zeigen die AutorInnen, dass die Vereinbarkeit von Arbeit und Privatleben als Mediator in der Beziehung zwischen Schichtarbeit und psychosomatischen Beschwerden agiert. Die vermittelnde Rolle von zeit- und belastungsbasierten Konflikten zwischen Berufs- und Privatleben wird auch im Beitrag von Reimann behandelt. Im Rahmen einer repräsentativen Stichprobe von Beschäftigten in Großbetrieben zeigt die Autorin, dass Beschäftige aufgrund von Belastungen durch die Arbeitsgestaltung größere Konflikte in der Kombination aus beruflichen und privaten Anforderungen erleben und dies wiederum negative Auswirkungen auf die psychische Gesundheit hat.

Das 6. Symposium der Arbeitszeitgesellschaft lieferte wieder spannende Einsichten in verschiedene Flexibili- sierungsaspekte, die sowohl die Verantwortung der Beschäftigten als auch der Arbeitgeberinnen und Arbeitgeber betreffen. Hierbei spielen strukturelle, soziale, wie auch persönliche Faktoren eine Rolle. Unter dem Motto „Innovationen in Forschung und Praxis“ trafen sich Mitglieder und Interessierte der Arbeitszeitgesellschaft auch in diesem Jahr in Wien. Hier wurden die neuesten Entwicklungen betrachtet und insbesondere die internationalen KonsensusPapiere der Working Time Society zu Schichtarbeit diskutiert.

Eine spannende Lektüre wünscht Ihnen

der Vorstand der Arbeitszeitgesellschaft e. V. 